\title{
Thirty years of European Conference on Spectroscopy of Biological Molecules celebrated in Ruhr University Bochum
}

\author{
Parvez I. Haris ${ }^{\mathrm{a}}$ and Klaus Gerwert ${ }^{\mathrm{b}}$ \\ ${ }^{\text {a }}$ Faculty of Health and Life Sciences, De Montfort University, The Gateway, Leicester, LE1 9BH, \\ United Kingdom \\ ${ }^{\mathrm{b}}$ Department of Biophysics, Ruhr University Bochum, Universitaetsstr. 150, 44801 Bochum, Germany
}

The 16th European Conference on Spectroscopy of Biological Molecules (ECSBM) was held at Ruhr University Bochum in Germany (6-10 September, 2015). The conference was chaired by Klaus Gerwert (see Fig. 1) and it coincided with the 30th year of this conference series. The conference is held in alternate years in different countries within Europe.

The first ECSBM was held at the University of Reims in September 1985. It was organised by Lucien Bernard (Rector Reims University) and his colleagues Alain Alix and Michel Manfait. In total three out of the 16th ECSBM conferences has been held in Germany and this reflects the large number of German scientists and institutions that focus on application of spectroscopy in biological research. The second ECSBM meeting was held in Freiburg, Germany, in September 1987 and after 18 years, in 2005, the conference returned back to Germany once more. The meeting was held than in Aschaffenburg. It is noteworthy that the 16th ECSBM conference in Bochum was a form of reunion for key scientists who were responsible for starting this conference series including Prof. Werner Mäntele (see Fig. 1). Werner, who is currently based at the Johann Wolfgang Goethe University Frankfurt am Main, was awarded the Life Time Achievement Award at the Bochum meeting for his outstanding contributions in the field of biological vibrational spectroscopy.

The conference in Bochum now (see Fig. 1) had a total of 222 participants from 26 different countries covering the continents of Europe, Asia and America. There were 69 lectures (15 invited lectures, 18 contributed talks and 36 selected talks from submitted abstracts).

This special issue of Biomedical Spectroscopy and Imaging presents a selection of work presented at the conference. It provides excellent examples of application of spectroscopic and imaging techniques for the study of diverse biological systems.

The conference in Bochum was dominated by impressive presentations which show how vibrational spectroscopy is used at different scales to solve biological questions. The talks demonstrated in a convincing manner how the use of time-resolved FTIR difference spectroscopy is able to determine molecular reaction mechanisms of proteins and protein interactions at the atomic level. For example the molecular reaction mechanisms of retinal-proteins like bacteriorhodopsin and channelrhodopsins and 


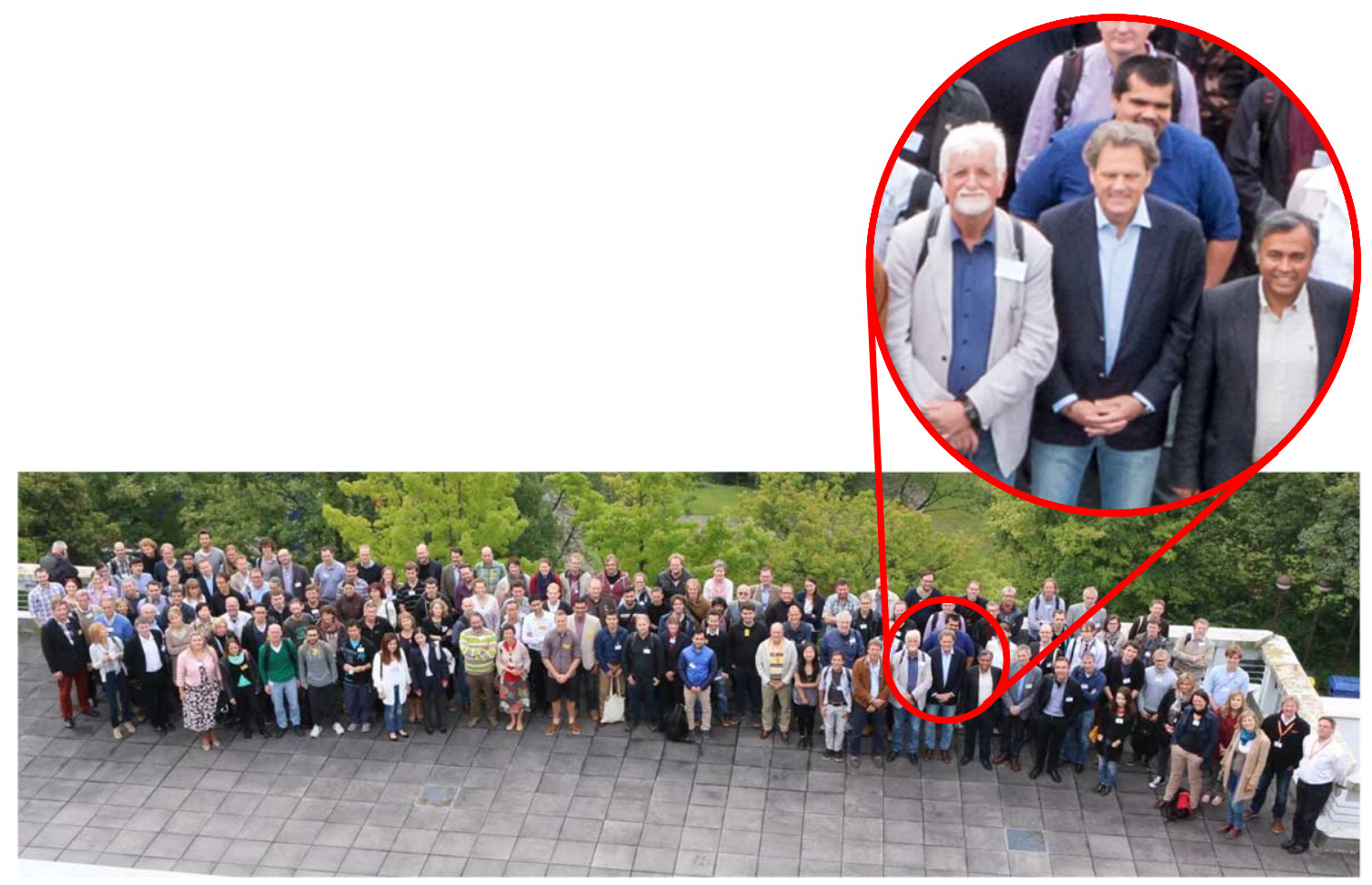

Fig. 1. Conference photograph (ECSBM 2015 - September 9th, 2015, Bochum, Germany). The caption shows the authors together with Life Time Achievement Award recipient Prof. Werner Mäntele.

of GTPases and ATPases, especially the catalysis of GTP hydrolysis by Ras were presented in several lectures.

Particularly outstanding, however, was the label-free imaging of tissues and cells by vibrational imaging techniques developed in recent years. For the imaging of individual cells, Raman spectroscopy is primarily presented, while tissue imaging predominantly utilises FTIR spectroscopy. The spatially-resolved vibrational spectra classify label-free cell organelles or diseased tissue components. Since tissue annotation is label-free, the method can be automated. Thereby cancer can be identified. This provides an important basis for treatment decisions by the clinician. However, Raman spectroscopy can do more than merely characterise cells: it can also be used to analyse the pharmacokinetics of therapeutically effective small molecules as presented further more in several talks.

It became obvious in the conference that there is a great potential and great future especially for vibrational spectroscopy in pharmaceutical and clinical applications. 